\title{
A percepção de universitários sobre as consequências do beber pesado episódico*
}

\section{Marília Ignácio de Espíndola \\ Daniela Ribeiro Schneider ${ }^{2}$ \\ Carolina Bunn Bartilott ${ }^{3}$}

* Apoio financeiro do Conselho Nacional de Desenvolvimento Científico e Tecnológico (CNPq), Brasil e da Universidade do Sul de Santa Catarina (UNISUL), Brasil.

${ }^{1}$ Universidade Federal de São Paulo, São Paulo, SP, Brasil.

${ }^{2}$ Universidade Federal de Santa Catarina, Florianópolis, SC, Brasil.

${ }^{3}$ Universidade do Sul de Santa Catarina, Tubarão, SC, Brasil.
Objetivo: verificar a percepção de universitários brasileiros sobre os riscos de um comportamento chamado de Beber Pesado Episódico (BPE). Método: estudo possui delineamento descritivo exploratório, de corte transversal, de natureza quantitativa, Participaram 2641 estudantes de todas as regiões do Brasil. Constatou-se que 24,8\% dos respondentes consideram-se um bebedor pesado. Resultados: verificou-se que a bebida mais utilizada na prática do BPE é a cerveja. Os homens praticaram mais o $\operatorname{BPE}(31,7 \%)$ do que as mulheres (19,5\%). Dos que já praticaram o BPE, 75,8\% responderam que repetiram esse consumo. Conclusão: uma parcela significativa dos universitários pratica o BPE e sua maioria tem uma baixa percepção do risco relacionado a esse comportamento.

Descritores: Bebedeira; Consumo de Álcool na Faculdade; Universidades; Abuso de Álcool.

\section{Como citar este artigo}

Espíndola MI, Schneider DR, Bartilott CB. The perception of college students about the consequences of binge drinking. SMAD, Rev Eletrônica Saúde Mental Álcool Drog. 2019;15(2):29-37 doi: https://dx.doi.org/ 


\title{
The perception of college students about the consequences of binge drinking
}

\begin{abstract}
Objetive: verify the perception of Brazilian university students about the risks of a behavior called Binge Drinking. Method: descriptive exploratory, cross-sectional, quantitative nature. Participated 2641 students from all regions of Brazil. It was found that $24.8 \%$ of respondents considered to be a heavy drinker. Results: it has been found that the beverage most used in the practice of binge drinking is beer. Those who have practiced the binge drinking, $75.8 \%$ responded that they repeated this consumption. Conclusion: is that a significant portion of university practices binge drinking and most do not realize the risk related to this behavior.
\end{abstract}

Descriptors: Binge Drinking; Alcohol Drinking in College; University; Universities; Alcohol Abuse.

\section{La percepción de los universitarios con respecto a las consecuencias de los episodios de consumo excesivo de alcohol}

\begin{abstract}
Objetivo: verificar la percepción de los estudiantes universitarios brasileños sobre los riesgos de un comportamiento llamado Episodios de consumo excesivo. Método: la investigación fue descriptiva-exploratoria, de corte transversal con naturaleza cuantitativa. Participaron 2641 estudiantes de todas las regiones de Brasil. Resultados: se constató que el $24,8 \%$ de los participantes se consideraban dentro del patrón del episodio de consumo excesivo y se verificó que la cerveza es la bebida más utilizada en dicha práctica. De los que ya habían realizado alguna vez el consumo excesivo, el 75,8\% respondieron que repitieron ese patrón. Conclusión: se concluye que un número significativo de universitarios practica los episodios de consumo excesivo de alcohol y que en su mayoría no perciben el riesgo relacionado a este comportamiento.
\end{abstract}

Descriptores: Borrachera; Consumo de Alcohol en la Universidad; Universidades; Abuso de Alcohol. 


\section{Introdução}

A vida universitária é caracterizada como um período de transformações e de acesso a um novo patamar da vida social e acadêmica/profissional, incluindo uma maior independência da família, a construção de novas amizades e a exigência da definição de novas perspectivas de vida. Por isso mesmo, dependendo da história de vida do estudante, este pode ser um período crítico, de muitas mudanças e desafios, o que os leva a serem, os universitários, um segmento em situação de maior vulnerabilidade e, portanto, de maior risco ao abuso de álcool e suas consequências ${ }^{(1-4)}$. Além disso, os hábitos culturais entre estes estudantes consolidam crenças normativas de que o beber é um comportamento comum em seu meio social, além da percepção compartilhada sobre a importância do beber na experiência de ser universitário(5) o que coloca no álcool uma espécie de instrumento de amálgama social. Sendo assim, comparado com outras populações em geral, esta categoria apresenta um padrão de consumo de álcool considerado de maior risco e realizado de forma mais frequente e intensa, o que justifica a necessidade de uma atenção específica para esse público ${ }^{(1,4,6)}$.

Uma parcela desses estudantes apresenta um comportamento chamado de Beber Pesado Episódico (BPE), termo proposto como alternativa de denominação, em português, para o fenômeno binge drinking(7-8). Conceituado como o consumo de elevada quantidade de álcool em um curto período de tempo ${ }^{(4,6,9)}$. Esse é um dos padrões de consumo mais perigosos e frequentementes e estão associado a uma série de problemas físicos e psicossociais ${ }^{(6,10)}$, sendo, por isso, considerado um problema de saúde pública(11).

Nos EUA Wechsler e Nelson ${ }^{(12)}$ foram os primeiros a apresentarem esse conceito, descrevendo-o como um padrão de consumo alcoólico popularizado entre os estudantes universitários americanos. O International Center for Alcohol Policies (ICAP)(7) informa que existem várias definições sobre as quantidades de doses consumidas pelo Bebedor Pesado Episódico e essa conceituação muda em relação à nacionalidade. A mais utilizada e adotada nessa pesquisa é apresentada pelo National Institute of Alcohol Abuse And Acoholism, [NIAAA] que a define como: "um "binge" é um padrão de beber álcool que em uma concentração sanguínea de $0,08 \mathrm{~g}$ por $100 \mathrm{ml}$ de álcool no sangue ou mais. Para um adulto típico esse padrão corresponde ao consumo de cinco ou mais doses* (masculino) ou quatro ou mais doses (femininos) em cerca de duas horas (tradução nossa)".

* Para comparação uma dose padrão de bebida alcoólica (350ml de cerveja, $150 \mathrm{ml}$ de vinho ou $50 \mathrm{ml}$ de destilado) contém, aproximadamente, $10-14 \mathrm{~g}$ de álcool puro (Arantes, 2012; Silveira et al., 2012).
É necessário considerar que essa definição se refere apenas aos episódios de compulsão individual e não contempla o número de ocorrências ou o período de tempo BPE e nem o padrão de consumo que pode acarretar sérias consequências. Para diferenciar a dependência de álcool do beber pesado episódico é necessário considerar o intervalo de tempo que esse consumo se repete. Alguns autores descrevem que esse intervalo pode ser de uma ou duas semanas, um mês, seis meses ou um ano(14). Esta pesquisa considerará a frequência de um mês.

Evidências científicas demonstram que essas quantidades de ingestão de álcool aumentam os riscos à exposição de problemas, tais como dependência, perda de consciência, risco de morte, infarto agudo do miocárdio, problemas gástricos, dentre outros ${ }^{(1,8,15-16)}$. Dentre as consequências comportamentais mais predominantes do BPE estão o envolvimento em acidentes de trânsito, prática de sexo desprotegido, gravidez indesejada, acometimento de doenças sexualmente transmissíveis, sofrer e/ou cometer abuso sexual, diminuição da produtividade acadêmica, envolvimento em brigas, prejuízo no progresso e estruturação de habilidades cognitivo-comportamentais ${ }^{(1,4,8-9,15-17)}$. Esse padrão de consumo está associado ao sono, performance acadêmica e retenção de conhecimentos ${ }^{(18)}$. Sendo assim, O BPE é considerado um comportamento de alto risco e está relacionado a diversas consequências negativas físicas, emocionais e sociais(1).

As maiores pesquisas científicas sobre os padrões de consumo de álcool provêm de países desenvolvidos. Nos EUA, desde 1993, os responsáveis pelas universidades americanas reconhecem o comportamento de binge drinking e suas consequências, por isso têm direcionado maior atenção a esses jovens e realizado levantamentos e pesquisas sobre os riscos nessa população jovem(12).

No II Levantamento Nacional sobre Álcool e Drogas, realizado em 2012, comparam-se dados com o I Levantamento Nacional Sobre os Padrões de Consumo de Álcool na População Brasileira, realizado em 2006(19), nos quais revelam a prevalência do Beber Pesado Episódico nos últimos 12 meses. No ano de 2006, 45\% dos sujeitos que se declaram não abstêmios afirmaram ocorrência de BPE em alguma vez nos últimos 12 meses, já em 2012 esse número subiu para 58\%, um aumento de $13 \%$. Entre os homens esse aumento foi de 12 pontos percentuais (54\% em 2006 e 66\%, em 2012). Entre as mulheres o crescimento foi um pouco maior em comparação aos homens e ao total: 14\% (34\% em 2006 e $48 \%$ em 2012)(19).

O governo federal também realizou um levantamento específico entre os universitários sobre padrão de uso de substâncias psicoativas. O "I Levantamento Nacional sobre o uso de álcool, Tabaco e outras drogas entre 
universitários das 27 Capitais Brasileiras", realizado com uma mostra de 12.673 participantes, descreve o comportamento do beber pesado e conclui que a cada quatro universitários brasileiros um apresenta pelo menos uma ocasião de beber pesado episódico em 30 dias anteriores à entrevista do levantamento e um em cada três afirmaram ter bebido nesse padrão nos últimos 12 meses $^{(1)}$. Sobre a prevalência do beber pesado entre universitários, 32,2\% informaram um padrão de consumo de mais de cinco doses em uma única ocasião numa frequência semanal e 1,9\% do total da amostra consumiu cinco ou mais doses em uma única ocasião quase todos os dias ${ }^{(1)}$.

Os universitários homens bebem em binge mais que as mulheres ${ }^{(1,8,10)}$ sendo que $31,3 \%$ deles já haviam feito uso de álcool no padrão BPE, em comparação com as mulheres, que corresponderam a $20,3 \%$ nos últimos 30 dias. Quando a período analisado considera os últimos 12 meses anteriores a pesquisa, a diferença é ainda mais evidente, $43,7 \%$ dos homens afirmaram ter bebido nesse padrão e $29 \%$ das mulheres.

Com relação à idade, o consumo tende a diminuir com o passar do tempo $(8,10)$. Entre jovens até 18 anos, 29\% já afirmaram ter bebido em binge nos 30 dias anteriores a pesquisa, $27,8 \% \mathrm{n}$ a faixa dos 18 e 24 anos; $22,9 \%$ entre $25-34$ anos e $17,8 \%$ são maiores de 35 anos $^{(1)}$. A pesquisa também identificou um elevado consumo entre universitários de Ciências Exatas (34,7\%) em comparação aos das áreas biológicas $(24,6 \%)$ e humanas $(23,3 \%)^{(1)}$.

O objetivo desse artigo é caracterizar o padrão de uso BPE entre universitários, assim como a sua percepção sobre as consequências do beber pesado episódico para sua vida acadêmica e social.

\section{Método}

Essa pesquisa teve delineamento descritivo exploratório, de corte transversal com natureza quantitativa observacional.

Para a coleta de dados foi utilizado um questionário construído a partir dos objetivos específicos dessa pesquisa. $O$ instrumento continha perguntas algumas perguntas abertas e outras fechadas. O questionário tinha ao todo 20 perguntas sendo onze relativas a dados de caracterização e perfil do sujeito, duas referentes ao padrão de consumo de álcool e cinco referentes à percepção e conhecimento acerca do BPE e seus riscos e consequências. Para essa análise foi utilizada apenas uma pergunta aberta sobre a frequencia mensal sendo que as demais foram questões fechadas.

A pesquisa foi feita a partir da inserção em grupos fechados de universitários de uma rede social da internet. Após a autorização dos moderadores era disponibilizado o instrumento no qual os participantes respondiam on-line. Ao todo foram 2641 respondentes, distribuídos em 311 Instituições de Ensino Superior (181 públicas e 144 privadas).

Foram utilizadas estatísticas descritivas e inferenciais, tomando como nível de significância estatística $5 \%(p<0,05)$, sendo a análise de consistência feita com teste $X^{2}$.

\section{Resultados}

Os participantes eram graduandos de diversas Instituições de Ensino Superior (IES) de todo o Brasil, totalizando 2641 participantes, sendo a média de idade de 22 anos $(S=5,2)$ em sua maioria de instituições públicas $(n=2188)$, estudantes de diversas áreas do conhecimento. Na Tabela 1 estão contidos os dados de caracterização geral dos sujeitos da pesquisa envolvendo sexo, idade, estado civil, se possui ou não atividade remunerada, religião, tipo de IES e as áreas do conhecimento dos respondentes.

Tabela 1 - Dados de caracterização dos respondentes. Brasil, 2016

\begin{tabular}{|c|c|c|c|}
\hline Variável & & N. & Percentual \\
\hline \multirow{2}{*}{ Sexo } & Feminino & 1480 & $56,0 \%$ \\
\hline & Masculino & 1161 & $44,0 \%$ \\
\hline \multirow{7}{*}{$\begin{array}{l}\text { Idade } \\
\text { (anos) }\end{array}$} & Menor ou igual a 18 & 332 & $12,6 \%$ \\
\hline & 19 até 24 & 1745 & $66,1 \%$ \\
\hline & 25 até 30 & 406 & $15,4 \%$ \\
\hline & 31 até 35 & 82 & $3,1 \%$ \\
\hline & 36 até 40 & 34 & $1,3 \%$ \\
\hline & 41 ou mais & 42 & $1,6 \%$ \\
\hline & Média & & \\
\hline \multirow{4}{*}{ Estado civil } & Solteiro(a) & 2351 & $89,0 \%$ \\
\hline & Casado(a) / União Estável & 264 & $10,0 \%$ \\
\hline & Divorciado(a)/ Separado(a) & 22 & $0,8 \%$ \\
\hline & Viúvo (a) & 4 & $0,2 \%$ \\
\hline \multirow{2}{*}{$\begin{array}{l}\text { Atividade } \\
\text { Remunerada }\end{array}$} & Não & 1385 & $52,4 \%$ \\
\hline & Sim & 1256 & $47,6 \%$ \\
\hline \multirow{6}{*}{ Moradia } & $\begin{array}{l}\text { Com os pais e/ou outros } \\
\text { parentes }\end{array}$ & 1502 & $56,9 \%$ \\
\hline & Com amigos/colegas & 612 & $23,2 \%$ \\
\hline & Sozinho & 299 & $11,3 \%$ \\
\hline & Com o cônjuge & 200 & $7,6 \%$ \\
\hline & Com Namorado (a) & 20 & $0,8 \%$ \\
\hline & Outros & 8 & $0,3 \%$ \\
\hline \multirow{7}{*}{ Religião } & $\begin{array}{l}\text { Agnóstico/ Ateu/ Sem } \\
\text { Religião }\end{array}$ & 928 & $35,1 \%$ \\
\hline & Católico & 830 & $31,4 \%$ \\
\hline & Evangélico/Protestante & 405 & $15,3 \%$ \\
\hline & Espírita & 275 & $10,4 \%$ \\
\hline & Deísta (Acredita em Deus) & 84 & $3,2 \%$ \\
\hline & Outras & 88 & $3,3 \%$ \\
\hline & Umbandista / Candoblé & 31 & $1,2 \%$ \\
\hline \multirow{2}{*}{ Tipo de IES* } & Pública & 2188 & $82,8 \%$ \\
\hline & Privada & 450 & $17,0 \%$ \\
\hline
\end{tabular}


Tabela 1 - continuação

\begin{tabular}{llcc}
\hline Variável & & N. & Percentual \\
\hline & Ciências Sociais Aplicadas & 570 & $21,6 \%$ \\
& Engenharias & 557 & $21,1 \%$ \\
& Ciências Humanas & 365 & $13,8 \%$ \\
Grande & Ciências da Saúde & 298 & $11,3 \%$ \\
Área do & Ciências Exatas e da Terra & 250 & $9,5 \%$ \\
Conhecimento & Outros & 157 & $5,9 \%$ \\
& Ciências Agrárias & 152 & $5,8 \%$ \\
& Lingüística, Letras e Artes & 150 & $5,7 \%$ \\
& Ciências Biológicas & 142 & $5,4 \%$ \\
\hline Total de & & & \multirow{2}{*}{2641} \\
Sujeitos & & &
\end{tabular}

Verifica-se na Tabela 1 que a maioria dos respondentes são do gênero feminino $(n=1480)$ perfazendo $56 \%$ do total. Referente a idade, predominaram os participantes de entre 19 e 24 anos $(66,1 \%)$ sendo a média de $22,5 \%$. A grande maioria dos respondentes é solteiro (89\%) e mora com os pais $(56.9 \%)$. Pouco mais da metade afirmaram não exercer alguma atividade remunerada (52,4\%). Quanto a religião ou denominação religiosa, teve-se a maior parte dos participantes afirmando-se ateus, agnóstico ou sem religião $(35,1 \%)$, sendo que depois predominam os católicos, e em menor proporção os evangélicos.

No que se refere ao tipo de instituição, $82,8 \%$ dos respondentes afirmaram que pertencem a instituição pública de ensino de ensino federal e estadual. A amostra de participantes foi classificada quanto a área de conhecimento da CAPES, sendo que os estudantes das Ciências Sociais Aplicadas tiveram o maior número de participação $(21,6 \%)$.

Sobre a percepção e o conhecimento do universitário sobre o BPE, 91,5\% dos participantes afirmaram que nunca haviam ouvido falar nesse padrão de consumo. Essa era uma das primeiras perguntas do questionário e no qual o respondente não havia recebido nenhuma informação ainda sobre o tema da pesquisa. A partir das informações da classificação da prática do BPE ( 5 doses para homens e 4 para mulheres em uma única ocasião) foi perguntado ao universitário se ele se considerava um Bebedor Pesado Episódico (Binge Drinker), sendo que $75,2 \%$ afirmaram que não se consideravam um BPE e $24,8 \%$ confirmaram considerar-se este tipo de bebedor.

Em relação à frequência do comportamento $\mathrm{BPE}$ entre os universitários respondentes, podemos observar os dados na Tabela 2.

Tabela 2 - Frequência do BPE* entre os respondentes. Brasil, 2016

\begin{tabular}{lcc}
\hline & Frequência & Porcentagem \\
\hline Em branco & 33 & 1,2 \\
Não houve repetição & 263 & 10,0 \\
No último mês & 581 & 22,0
\end{tabular}

(continua...)
Tabela 2 - continuação

\begin{tabular}{lcc}
\hline & Frequência & Porcentagem \\
\hline Nos últimos 3 meses & 473 & 17,9 \\
\hline Nos últimos 6 meses & 356 & 13,5 \\
Nos últimos 12 meses & 592 & 22,4 \\
Nunca aconteceu esse tipo & 343 & 13,0 \\
de consumo & 2641 & 100,0 \\
Total &
\end{tabular}

Nessa pesquisa buscou-se analisar em que frequência a pratica do BPE se repetia entre os universitários. Destaca-se que $85,80 \%$ já tiveram algum episódio de binge em algum momento de sua vida, sendo $75,80 \%$ esse episódio ocorreu no último ano. Um índice significativo, inclusive, indicou ter consumido em BPE no último mês $(22,0 \%)$, indicando nesse caso um padrão maior de risco. Interessante notar a discrepância entre a percepção de si e o padrão de uso, pois apesar de somente $24,8 \%$ se considerarem um bebedor pesado episódico, 39,90\% da amostra teve episódios binge nos últimos três meses.

Na sequência foi verificada a quantidade de doses ingeridas por ocasião de diferentes tipos de bebidas, conforme se verifica na Tabela 3 .

Tabela 3 - Frequência mensal e tipos de bebidas alcóolicas mais consumidas em BPE* entre universitários. Brasil, 2016

\begin{tabular}{lccc}
\hline & $\begin{array}{c}\text { Inferior a 5 } \\
\text { doses }\end{array}$ & 5 a 10 doses & $\begin{array}{c}\text { Acima de 11 } \\
\text { doses }\end{array}$ \\
\hline Cerveja ou chopp & $83,3 \%$ & $12,4 \%$ & $4,1 \%$ \\
Vinho & $96,8 \%$ & $2,6 \%$ & $0,6 \%$ \\
Bebida tipo Ice & $98,2 \%$ & $1,5 \%$ & $0,2 \%$ \\
Bebidas destiladas & $91,1 \%$ & $7,2 \%$ & $1,6 \%$ \\
*BPE: Beber Pesado Episódico & &
\end{tabular}

Verificou-se que a bebida utilizada com maior frequência e intensidade pelos universitários brasileiros em situações de binge é a cerveja, em relação a qual $12,4 \%$ afirmaram que beberam de 5 a 10 doses em uma única ocasião e 4,1\% afirmaram que beberam acima de 11 doses. Em segundo lugar no BPE está o uso de bebidas destiladas.

A seguir apresenta-se a associação entre variáveis, utilizado o teste $X^{2}$ de Person. Destaca-se aqui os resultados que apresentaram relevância significativa, como se verifica na Tabela 4.

Os dados corroboram achados anteriores, como vimos acima, em que os homens consideram estar em um padrão BPE $(31,7 \%)$ mais do que as mulheres.

As faixas etárias que mais se reconhecem no padrão de consumo BPE são as mais jovens, nas faixas etárias de 19 à 24 anos (26,4\%) e dos 25 a 30 (26,6\%) juntas correspondem a metade da amostra. Referente ao estado civil os universitários solteiros são os que apresentaram a maior incidência desse comportamento $(26,1 \%)$, proporção maior do que os $15 \%$ dos casados 
afirmaram que se consideram BPE. Quanto a moradia os participantes que moram com amigos ou sozinhos apresentaram maior correlação $(32,4$ e $30,4 \%$ respectivamente) de uso neste padrão.

Interessante destacar também a relação com a religião, sendo que os estudantes que se consideram agnósticos/ateus/sem religião tem maior incidência de padrão BPE (33,5\%).

As áreas do conhecimento de Engenharias e das Ciências Sociais Aplicadas apresentaram a maior prevalência nessa categoria: $30 \%$ e $29,6 \%$ respectivamente.

Tabela 4 - Correlações entre os dados do perfil e o consumo no padrão BPE entre universitários. Brasil, 2016

\begin{tabular}{|c|c|c|c|c|c|c|c|}
\hline & \multirow[t]{2}{*}{ Variável } & \multirow[t]{2}{*}{ N. } & \multicolumn{2}{|c|}{$\begin{array}{l}\text { Se considera um } \\
\text { Binge Drinking? }\end{array}$} & \multirow[t]{2}{*}{$\begin{array}{l}\text { Percentual } \\
\text { "Binge" }\end{array}$} & \multirow[t]{2}{*}{$X^{2 *} e G^{\dagger}$} & \multirow[t]{2}{*}{$\begin{array}{l}\text { Significância } \\
\text { Estatística }\end{array}$} \\
\hline & & & NÃO & SIM & & & \\
\hline \multirow{2}{*}{ Sexo } & Masculino & 1480 & 1192 & 288 & $31,7 \%$ & \multirow{2}{*}{$\mathrm{X}^{2 *}=52,1 \mathrm{gl}^{\dagger}=1$} & \multirow{2}{*}{$p=0,000$} \\
\hline & Feminino & 1161 & 793 & 368 & $19,5 \%$ & & \\
\hline \multirow{6}{*}{$\begin{array}{l}\text { Idade } \\
\text { (anos) }\end{array}$} & Menor ou igual a 18 & 332 & 267 & 65 & $19,6 \%$ & \multirow{6}{*}{$\mathrm{X}^{2 *}=19,\left.49 \mathrm{~g}\right|^{\dagger}=5$} & \multirow{6}{*}{$p=0,002$} \\
\hline & 19 até 24 & 1745 & 1284 & 461 & $26,4 \%$ & & \\
\hline & 25 até 30 & 406 & 298 & 108 & $26,6 \%$ & & \\
\hline & 31 até 35 & 82 & 68 & 14 & $17,1 \%$ & & \\
\hline & 36 até 40 & 34 & 29 & 5 & $14,7 \%$ & & \\
\hline & 41 ou mais & 42 & 39 & 3 & $7,1 \%$ & & \\
\hline \multirow{4}{*}{ Estado civil } & Solteiro(a) & 2351 & 1738 & 613 & $26,1 \%$ & \multirow{4}{*}{$\mathrm{X}^{2 *}=17,9 \mathrm{gl}^{\dagger}=3$} & \multirow{4}{*}{$p=0,000$} \\
\hline & Casado(a) / União Estável & 264 & 224 & 40 & $15,2 \%$ & & \\
\hline & Divorciado(a)/ Separado(a) & 22 & 19 & 3 & $13,6 \%$ & & \\
\hline & Viúvo (a) & 4 & 4 & 0 & $0,0 \%$ & & \\
\hline \multirow{2}{*}{$\begin{array}{l}\text { Atividade } \\
\text { Remunerada }\end{array}$} & Não & 1385 & 1050 & 335 & $24,2 \%$ & \multirow{2}{*}{$\mathrm{X}^{2 *}=0,6 \mathrm{gl}^{\dagger=1}$} & \multirow{2}{*}{$p=0,41$} \\
\hline & Sim & 1256 & 935 & 321 & $25,6 \%$ & & \\
\hline \multirow{6}{*}{ Moradia } & Com amigos/colegas & 612 & 414 & 198 & $32,4 \%$ & \multirow{6}{*}{$\mathrm{X}^{2 *}=43,8 \mathrm{gl}^{\dagger}=5$} & \multirow{6}{*}{$p=0,000$} \\
\hline & Sozinho & 299 & 208 & 91 & $30,4 \%$ & & \\
\hline & Com os pais e/ou outros parentes & 1502 & 1166 & 336 & $22,4 \%$ & & \\
\hline & Com Namorado (a) & 20 & 17 & 3 & $15,0 \%$ & & \\
\hline & Com o cônjuge & 200 & 173 & 27 & $13,5 \%$ & & \\
\hline & Outros & 8 & 7 & 1 & $12,5 \%$ & & \\
\hline \multirow{7}{*}{ Religião } & Agnóstico/ Ateu/ Sem Religião & 928 & 617 & 311 & $33,5 \%$ & \multirow{7}{*}{$\mathrm{X}^{2 *}=75,9 \mathrm{gl}^{\dagger}=6$} & \multirow{7}{*}{$p=0,000$} \\
\hline & Católico & 830 & 647 & 183 & $22,0 \%$ & & \\
\hline & Evangélico/Protestante & 405 & 350 & 55 & $13,6 \%$ & & \\
\hline & Espírita & 275 & 224 & 51 & $18,5 \%$ & & \\
\hline & Outras & 88 & 61 & 27 & $30,7 \%$ & & \\
\hline & Deísta (Acredita em Deus) & 84 & 62 & 22 & $26,2 \%$ & & \\
\hline & Umbandista / Candoblé & 31 & 24 & 7 & $22,6 \%$ & & \\
\hline \multirow{9}{*}{$\begin{array}{l}\text { Grande Área do } \\
\text { Conhecimento }\end{array}$} & Engenharias & 557 & 390 & 167 & $30,0 \%$ & \multirow{9}{*}{$\mathrm{X}^{2 *}=27,0 \mathrm{gl}^{\dagger}=8$} & \\
\hline & Ciências Sociais Aplicadas & 570 & 401 & 169 & $29,6 \%$ & & \\
\hline & Ciências Exatas e da Terra & 250 & 193 & 57 & $22,8 \%$ & & \\
\hline & Ciências Agrárias & 152 & 119 & 33 & $21,7 \%$ & & \\
\hline & Ciências da Saúde & 298 & 234 & 64 & $21,5 \%$ & & $p=0,001$ \\
\hline & Lingüística, Letras e Artes & 150 & 118 & 32 & $21,3 \%$ & & \\
\hline & Outros & 157 & 125 & 32 & $20,4 \%$ & & \\
\hline & Ciências Humanas & 365 & 291 & 74 & $20,3 \%$ & & \\
\hline & Ciências Biológicas & 142 & 114 & 28 & $19,7 \%$ & & \\
\hline Total de Sujeitos & & & & & & & 2641 \\
\hline
\end{tabular}

Verificou-se os locais mais comuns em que os universitários costumam consumir bebidas alcoólicas, estabelecendo uma comparação com a auto percepção desse padrão de consumo (Figura1). Destaca-se que o número dos que afirmaram que o padrão de consumo ocorre no Campus Universitário ou nas Imediações do Campus, ou ainda em festas de forma geral, espaços com forte relação com o comportamento de binge drinking. Necessário considerar que os respondentes poderiam informar mais de uma resposta. 


\section{Locais de Ocorrência BPE}

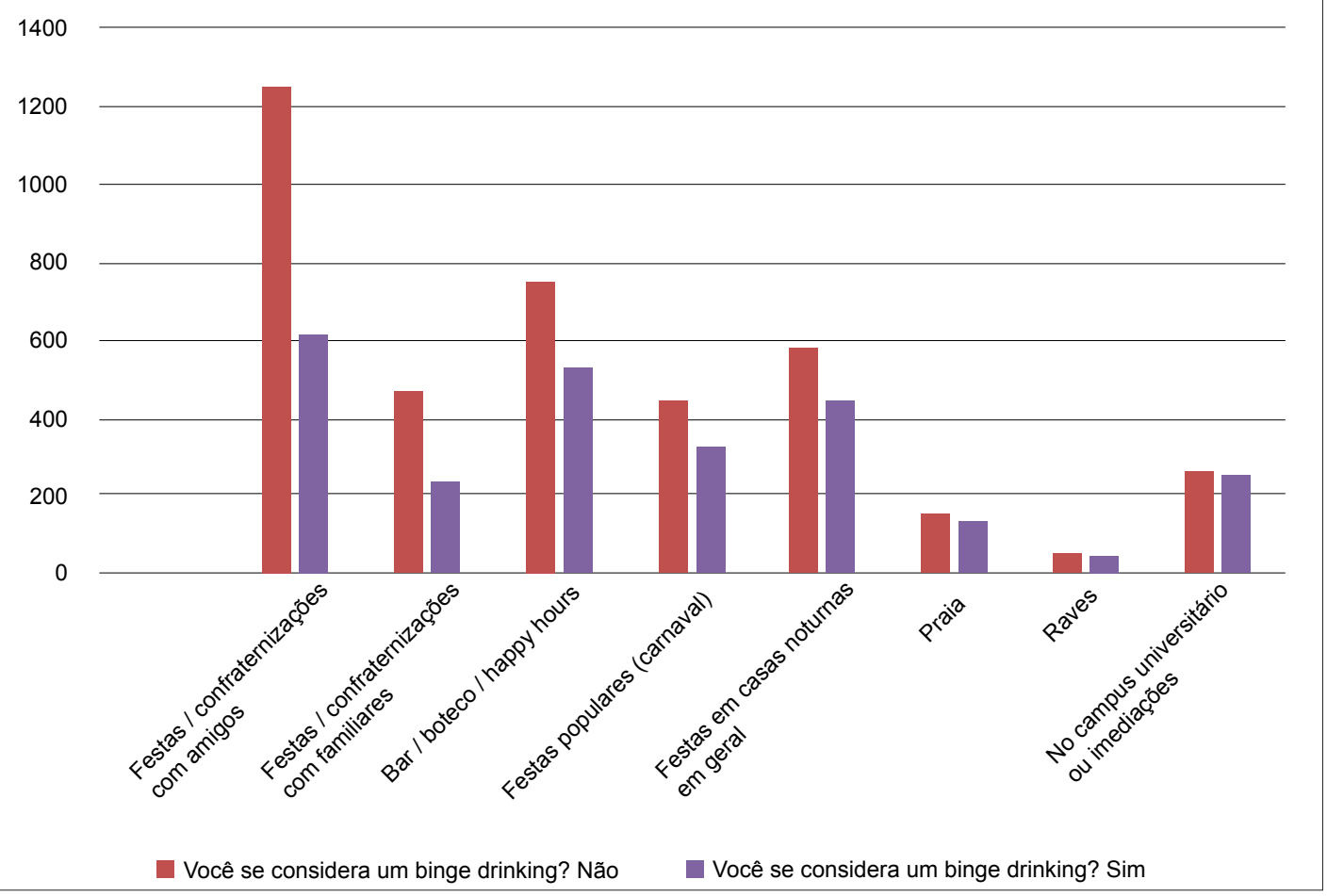

Figura 1 - Locais de maior ocorrência de Beber Pesado Episódico entre universitários. Brasil, 2016

Outras informações importantes dizem respeito à percepção do risco do comportamento BPE 48,7\% responderam que esse padrão de consumo é de alto risco para saúde, $7,4 \%$ afirmaram que é de médio risco e $43,9 \%$ disseram que é de baixo risco para a saúde. Cabe afirmar que essa pergunta foi feita no final do questionário no qual os estudantes já haviam recebido algumas informações sobre o comportamento BPE.

\section{Discussão}

Comparando os dados da literatura de referência na área com os resultados dessa pesquisa pode-se confirmar que o comportamento de beber pesado episódico ou binge drinking tem sido muito comum em universitários brasileiros, assim como se constata em outros países como os $\operatorname{EUA}^{(4,9,12)}$.

Confirma-se também que os homens bebem mais em binge do que as mulheres, corroborando com os resultados de levantamentos nacionais ${ }^{(1,19)}$ e com outras pesquisas ${ }^{(8)}$, ainda que haja um indicativo de um progressivo aumento desse comportamento entre mulheres ${ }^{(19)}$.

Deve-se salientar que nesse trabalho os respondentes demonstraram não conhecer o conceito de BPE ou binge drinking, já que 96,4\% nunca havido ouvido falar nesse padrão de consumo, apesar que $24,8 \%$ considera-se um bebedor pesado episódico depois de explicado o seu significado, e que $85,80 \%$ respondeu já ter tido ocasião de uma experiência de beber pesado episódica. Tal consideração nos remete à necessidade de problematizar esse tema com os universitários, já que nas perguntas finais do instrumento utilizado na presente pesquisa (após terem algumas informações sobre o que é esse padrão de consumo), 51,4\% afirmaram que esse tipo de consumo alcoólico é de médio ou baixo risco a saúde. Os estudantes universitários subestimam aos efeitos negativos do álcool e, em especial, do beber pesado episódico, o que consequentemente, leva-os a uma facilidade de exposição de situações de riscos e prejuízos a sua saúde ${ }^{(9)}$.

Apesar se serem significativas as investigações sobre o BPE, poucos pesquisadores têm focado especificamente em como os estudantes universitários pensam sobre esse padrão de consumo. Aumentar o entendimento sobre a forma que os estudantes universitários abordam e definem uso de álcool é importante para aumento de técnicas e ações preventivas $^{(18)}$.

No I Levantamento Nacional sobre o uso de álcool, tabaco e outras drogas entre universitários das 27 Capitais brasileiras identificou-se um elevado consumo entre universitários de Ciências Exatas com o comportamento BPE $(34,7 \%)^{(1)}$. Nesse presente estudo os estudantes foram classificados de forma mais detalhada em comparação ao Levantamento mencionado, que utilizou somente as áreas de estudo 
(Biológicas, Humanas e Exatas). Entretanto, os resultados foram semelhantes, quanto a incidência desse comportamento, já que no presente estudo as Engenharias obtiveram uma prevalência na percepção dos universitários sobre o BPE (30\%), seguida pelas Ciências Sociais Aplicadas (29,8\%) e em terceiro as Ciências Exatas e da Terra (22,8\%). Podemos afirmar que esses dados podem ter influência com a questão de gênero, considerando que o BPE é mais comum em homens e nos cursos de engenharias e das Ciências Exatas há uma predominância masculina.

Outro dado importante a ser discutido nesse artigo é referente à frequência de ingestão de bebida alcoólica. Esse estudo revelou que os participantes quando praticam o BPE, utiliza mais a cerveja, no qual $16,5 \%$ afirmaram beber acima de 5 doses em uma única ocasião. Esta pode ser a bebida mais consumida em função de ter preço mais acessível, muita propaganda ainda permitida sobre ela, além de ser culturalmente muito bem aceita em nosso país. Em segundo lugar estão os destilados como bebida predileta do universitário para pratica do BPE $(8,8 \%)$. Esses dados são semelhantes aos do II LENAD ${ }^{(19)}$ no qual relatam que a ingestão de cerveja e destilados, em indivíduos adultos, são as prediletas quando o consumo é em grande quantidade.

$O$ perfil de estudante de maior risco de praticar o BPE é o de homens, até 24 anos, solteiros, morando com amigos ou sozinho e que são agnóstico/ateu/não religiosos. Quem consome álcool nas imediações da universidade tem um risco maior de tomar em binge. Esses são indicadores importantes para a formulação de projetos de prevenção e de redução de danos ao beber pesado episódico.

Essa pesquisa foi além ao levar em consideração repetição dessa frequência (Tabela 2 ), já que é um dado que o próprio NIAA não fornece ${ }^{(8)}$. Dos que afirmaram ter praticado o BPE $75,8 \%$ responderam que repetiram esse consumo. Os riscos de desencadear dependência alcoólica e de sofrer os efeitos negativos do álcool aumentam a medida que a frequência de intoxicação por BPE se repetem ${ }^{(4)}$.

Os fatores mais protetivos que apareceram foram ser mais velho, estar casado e morar com a companheira/esposa ou com os pais. A prática da religião, principalmente a evangélica e espírita, também se coloca como um fator de proteção ao abuso do álcool entre os estudantes, assim como demostram outros estudos relacionados aos comportamentos de uso de drogas $^{(21)}$.

\section{Conclusão}

Essa pesquisa reforçou os achados de que os universitários brasileiros têm um padrão significativo de pratica do BPE e uma parcela significativa tem uma baixa percepção dos riscos envolvidos nesse comportamento. Devido a popularização do termo binge drinking pelos pesquisadores na década de 1990 o termo também se popularizou na mídia, mas que há pouca preocupação com as percepções dos estudantes que praticam o $\mathrm{BPE}$, especialmente relativo a definição do termo. Essa pesquisa vem demostrar o quanto ainda precisamos avançar relativo ao conhecimento dos universitários quanto ao comportamento de BPE.

Dados demonstraram que os universitários são uma população vulnerável devido ao alto consumo e frequência do uso de álcool. Esse estudo demonstrou que as quantidades de bebida alcoólica que essa população ingere são altas se comparadas com outras categorias sociais. As doses consumidas por esses estudantes e nessa frequência, podem acarretar sérios prejuízos à saúde em médio e a longo prazo.

Constata-se que nas universidades que esse comportamento é muito estimulado nas propagandas de festas universitárias, muitas delas em estilo "open bar" e em shows específicos a esse público, além dos próprios trotes universitários, que introduzem os calouros no que eles costumam chamar de "circuito etílico", tratando a questão do uso do álcool como algo naturalizado e como parte do ser universitário. Os próprios empresários exploram essa tendência ao abrir muitos bares ao redor dos campus universitários, com promoções de bebidas a preços mais acessíveis e, por isso mesmo, consumidas em maior quantidade, facilitando o acesso ao álcool e a prática de BPE, reforçando ainda mais a necessidade de uma atenção maior a esses estudantes. Esse estudo mostra que é necessário ter um olhar atento a população universitária quanto as consequências do BPE, pois quando eles procuram os dispositivos de saúde essas consequências podem já estar comprometendo a vida desse estudante de forma mais séria.

O presente estudo serve de reforço para que as autoridades brasileiras, reitorias e departamentos de assistência estudantil das universidades desenvolvam políticas preventivas e de redução de danos ao beber pesado episódico nos ambientes universitários.

Este estudo teve como uma de suas limitações ter utilizado um questionário próprio e não um já validado por outras pesquisas, que permitiria uma maior comparabilidade dos dados. É necessário desenvolver mais pesquisas e estudos, que complementem os dados, principalmente com viéses mais qualitativos, visando o descortinar dos sentidos do BPE entre universitários, na medida em que a percepção de riscos nesta pesquisa foi levantada a partir de dados somente quantitativos, o que perde a riqueza do conteúdo perceptivo. 


\section{Referências}

1. Andrade AG, Duarte PCAV, Oliveira LG. I levantamento nacional sobre o uso de álcool, tabaco e outras drogas entre universitários das 27 capitais brasileiras. Brasília: Secretaria Nacional de Políticas sobre Drogas; 2010.

2. Cardoso FM, Barbosa HA, Costa FM da, Vieira MA, Caldeira AP. Fatores associados à prática do binge drinking entre estudantes da área da saúde. Rev CEFAC. [Internet]. 2015 Apr [cited 2017 Mar7] ;17(2):475-84. Available from: http://www.scielo.br/scielo.php?script=sci_art text\&pid $=\mathrm{S} 151618462015000200475 \& \mathrm{Ing}=\mathrm{en}$. http://dx.doi.org/10.1590/1982-0216201589143

3. Hingson RW, Zha W. Age of Drinking Onset, Alcohol Use Disorders, Frequent Heavy Drinking, and Unintentionally Injuring Oneself and Others After Drinking. Pediatrics. 2009;123(6):1477-84.

4. Peuker AC, Fogaça J, Bizarro L. Expectativas e beber problemático entre universitários. Psicologia: teoria e pesquisa. 2006;22(2):193-200.

5. Hustad JT, Pearson MR, Neighbors C, Borsari B. The role of alcohol perceptions as mediators between personality and alcohol-related outcomes among incoming collegestudent drinkers. Psychol Addict Behav. 2014;28(2):336. 6. Knight R, Norman P. Impact of brief self-affirmation manipulations on university students' reactions to risk information about binge drinking. $\mathrm{Br} \mathrm{J}$ Health Psychol. 2016 Sep;21(3):570-83. doi: 10.1111/bjhp.12186.

7. Book IIB. Practical guides for alcohol policy and prevention approaches. Module. 2005;11:101-3.

8. Silveira CM, Silveira CC, Silva JGd, Silveira LM, Andrade AGd, Andrade LHSGd. Epidemiologia do beber pesado e beber pesado episódico no Brasil: uma revisão sistemática da literatura. Rev Psiquiatr Clín. 2008;35(suppl 1):31-8.

9. De Andrade AG, Silveira CM. Problemas comportamentais ligados ao uso de álcool. Rev USP. 2013(96):7-22.

10. Floripes TMF. Beber se embriagando (binge drinking): estudo de uma população de estudantes universitários que fazem uso do álcool de risco. [dissertação]. Botucatu (SP): Universidade Estadual Paulista Júlio de Mesquita Filho; 2008. 102 p.

11. Davoren MP, Demant J, Shiely F, Perry IJ. Alcohol consumption among university students in Ireland and the United Kingdom from 2002 to 2014: a systematic review. BMC Public Health. 2016;16(1):173.

12. Wechsler $H$, Nelson TF. Binge drinking and the American college students: What's five drinks? Psychol Addict Behav. 2001;15(4):287.

13. National Advisory Council on Alcohol Abuse and Alcoholism (NIAAA). Consideration of Recommendation on Definition of Binge Drinking. [Internet]. Summary of the 105th Meeting February 4-5, 2004 [Acesso 19 abr 2017]. Disponível em: https://pubs.niaaa.nih.gov/ publications/StrategicPlan/NIAAASTRATEGICPLAN. htm\#Drinking_Patterns

14. Courtney KE, Polich J. Binge drinking in young adults: Data, definitions, and determinants. Psychological bulletin. 2009;135(1): 142 .

15. Cavariani MB, de Oliveira JB, Kerr-Corrêa F, Lima MCP. Expectativas positivas com o uso de álcool e o beber se embriagando: diferenças de gênero em estudo do Projeto GENACIS, São Paulo, Brasil. Cad Saúde Pública. 2012:1394-404.

16. Naimi TS, Brewer RD, Mokdad A, Denny C, Serdula MK, Marks JS. Binge drinking among US adults. JAMA. 2003;289(1):70-5.

17. Arantes LFR. Binge drinking: um estudo bibliométrico (1999-2010) dos artigos publicados na base de dados SciELO. Estud Psicol. (Campinas). 2012:253-7.

18. Clinkinbeard SS, Johnson MA. Perceptions and practices of student binge drinking: an observational study of residential college students. Journal of drug education. 2013;43(4):30119.

19. Laranjeira R, Madruga C, Ribeiro M, Pinsky I, CaetanoR, Mitsuhiro S. II LENAD-Levantamento Nacional de Álcool e Drogas. São Paulo: Instituto Nacional de Ciência e Tecnologia para Políticas Públicas do Álcool e Outras Drogas; Universidade Federal de São Paulo; 2012.

20. Silveira CM, Siu ER, Wang Y-P, Viana MC, Andrade AGd, Andrade LH. Gender differences in drinking patterns and alcohol-related problems in a community sample in São Paulo, Brazil. Clinics. 2012;67(3):205-12. 21. Sanchez ZVDM, Nappo SA. A religiosidade, a espiritualidade e o consumo de drogas. Rev Psiquiatr Clín.2007;34(suppl 1):73-81. doi:http://dx.doi. org/10.1590/S0101-60832007000700010.

Recebido: 19.04.2017 Aceito: 01.11 .2018 